\title{
The association between the use of ivermectin and mortality in patients with COVID-19: a meta-analysis
}

\author{
Chia Siang Kow ${ }^{1} \cdot$ Hamid A. Merchant ${ }^{2} \cdot$ Zia UI Mustafa $^{3} \cdot$ Syed Shahzad Hasan ${ }^{2,4}$
}

Received: 6 January 2021 / Revised: 27 February 2021 / Accepted: 6 March 2021 / Published online: 29 March 2021

(c) Maj Institute of Pharmacology Polish Academy of Sciences 2021

\begin{abstract}
Objective The effect of ivermectin on mortality in patients with novel coronavirus disease 2019 (COVID-19) has been investigated in many studies. We aimed to perform a meta-analysis of randomized controlled trials to investigate the overall effect of ivermectin on the risk of mortality in patients with COVID-19.

Methods We systematically searched PubMed, Cochrane Central Register of Controlled Trials, Google Scholar, and preprint repository databases (up to February 28, 2021). Random-effects and inverse variance heterogeneity meta-analysis were used to pool the odds ratio of individual trials. The risk of bias was appraised using Version 2 of the Cochrane risk-of-bias tool for randomized trials.

Results Six randomized controlled trials were included in this analysis with a total of 658 patients who were randomized to receive ivermectin and 597 patients randomized in the control group who did not receive ivermectin. Of six trials, four had an overall high risk of bias. The estimated effect of ivermectin indicated mortality benefits (pooled odds ratio $=0.21$; 95\% confidence interval $0.11-0.42, n=1255$ ), with some evidence against the hypothesis of 'no significant difference' at the current sample size.

Conclusion We observed a preliminary beneficial effect on mortality associated with ivermectin use in patients with COVID19 that warrants further clinical evidence in appropriately designed large-scale randomized controlled trials.
\end{abstract}

Keywords Bias $\cdot$ Clinical trial $\cdot$ COVID-19 $\cdot$ Ivermectin $\cdot$ Mortality

\section{Introduction}

Ivermectin is well known for its efficacy against a range of parasitic diseases, including onchocerciasis and strongyloidiasis. Recently, a systematic review [1] of in vivo and in vitro studies of ivermectin demonstrated its broad antiviral activity against a range of RNA and DNA viruses. Particularly, ivermectin demonstrated an in vitro antiviral effect towards the severe acute respiratory syndrome coronavirus

Chia Siang Kow

chiasiang_93@hotmail.com

1 School of Postgraduate Studies, International Medical University, Kuala Lumpur, Malaysia

2 School of Applied Sciences, University of Huddersfield, Huddersfield, UK

3 Department of Pharmacy, District Headquarters Hospital, Pakpattan, Pakistan

4 School of Biomedical Sciences and Pharmacy, University of Newcastle, Callaghan, Australia
2 (SARS-CoV-2) [2]. This has since attracted the interest of frontline clinicians to investigate the potential repurposing of ivermectin for the treatment of coronavirus disease 2019 (COVID-19). Therefore, we aimed to perform a meta-analysis of randomized clinical trials to investigate the mortality benefits of ivermectin in patients with COVID-19.

\section{Methods}

We performed a systematic literature search in electronic databases including PubMed, Google Scholar, Cochrane Central Register of Controlled Trials, and preprint servers (medRxiv, Research Square, SSRN) with no language restriction, for eligible studies published up to February 28,2021 . The search strategy was built based on the following keywords and MeSH terms: "COVID-19", "SARS-CoV-2", and "ivermectin". The clinical trial registries of the United States (clinicaltrials.gov), China (chictr.org.cn), and World Health Organization (who.int/ 
clinical-trials-registry-platform) were also searched for registered clinical trials of ivermectin for the treatment of COVID-19 to identify studies with reported findings. Two investigators (CSK and $\mathrm{SSH}$ ) independently performed the literature screening to identify eligible studies. The reference lists of relevant articles were also reviewed to search for additional studies. Studies eligible for inclusion were randomized controlled trials comparing the mortality outcomes of ivermectin and its comparators in patients with COVID-19. We excluded studies with observational design, single-arm trials, non-randomized trials, and trials that did not report clinical outcomes. The systematic review followed the Preferred reporting Items for Systematic Reviews and Meta-Analyses (PRISMA) guidelines [3].

The outcome of interest was all-cause mortality. Each included trial was independently evaluated by two investigators (CSK and SSH) who also extracted the study characteristics. Data collected included author(s), trial design, country, patients' age, regimen of ivermectin, regimen of comparator treatment, and mortality outcomes. Two investigators (CSK and SSH) assessed the risk of bias of the trials included with Version 2 of the Cochrane risk-of-bias tool for randomized trials (RoB 2) [4], which is a standardized method for assessing potential bias in reports of randomized interventions. RoB 2 is structured into a fixed set of domains of bias, focusing on different aspects of trial design, conduct, and reporting. We used both the random-effects model and inverse variance heterogeneity model for the meta-analysis to estimate the pooled odds ratio at $95 \%$ confidence intervals. We examined the heterogeneity between studies using the $I^{2}$ statistics and the $\chi^{2}$ test at $50 \%$ and $P<0.10$, respectively. All analyses were performed using Meta XL, version 5.3 (EpiGear International, Queensland, Australia).

\section{Results}

Our systematic literature search retrieved 512 hits of which 401 were unique (titles retrieved after removing duplications) (Fig. 1). After screening, six randomized controlled trials [5-10] were included with a total of 658 patients who were randomized to receive ivermectin, and 597 patients were randomized to the control group who did not receive ivermectin. The six randomized trials that were included in the meta-analysis were respectively from Iraq [5], Iran [6], Bangladesh [7], Egypt [8], Turkey [9], and India [10]. Details of the included studies are shown in Table 1. A differing dosage regimen of ivermectin was noted across the six randomized controlled trials (Table 1).

The overall risk of bias assessed by RoB 2 is presented in Table 1. The trial by Niaee et al. [6] and Ravikirti et al. [10] had an overall low risk of bias. The other trials had an overall high risk of bias; the trial led by Mahmud et al. [7] had a high risk of bias in the domain of missing outcome data since a significant proportion $(21 \%)$ of participants randomized was not included in the analysis; the trial by Elgazzar et al. [8] had a high risk of bias in the domain of randomization since the protocol for randomization was not reported; the trial by Hashim et al. [5] had a high risk of bias in the domain of randomization due to simple randomization method used with no information on allocation concealment and had also some concerns of bias in the domain of deviations from intervention due to unblinded participants; the trial led by Okumuş et al. [9] had a high risk of bias in the domain of randomization due to simple randomization method used and had also a high risk of bias in the domain of deviations from intended interventions due to open-label nature of the trial. The aforementioned four trials [5, 7-9] had a low risk of bias for other domains assessed.

The meta-analysis revealed a significantly reduced odds of mortality with the use of ivermectin among patients with COVID-19 relative to non-use of ivermectin; the estimated effect of ivermectin indicated mortality benefits (Fig. 2; pooled odds ratio $=0.21 ; 95 \%$ confidence interval 0.11 to $0.42, n=1255$ ) and with some evidence against the hypothesis 'no significant difference' at the current sample size.

Some may be concerned by the absence of real-time reverse transcription-polymerase chain reaction confirmatory test for COVID-19 diagnosis in a significant fraction of subjects in the trial by Niaee et al. [6], but exclusion of this trial in the meta-analysis still observed a significantly reduced risk of mortality with the use of ivermectin among patients with COVID-19 relative to non-use of ivermectin (pooled odds ratio $=0.23 ; 95 \%$ confidence interval 0.09-0.56).

\section{Discussion}

The mortality benefits demonstrated with the use of ivermectin in patients with COVID-19 from this meta-analysis is in agreement with the findings from the recent systematic review [1] and the largest observational study [11] on ivermectin thus far exhibiting a significantly reduced odds of mortality associated with the use of ivermectin (odds ratio $=0.27 ; 95 \%$ confidence interval $0.09-0.80)$. We, therefore, conclude a preliminary positive effect on mortality associated with ivermectin use in hospitalized patients with COVID-19 that warrants further clinical evidence in appropriately designed large-scale randomized controlled trials. Nevertheless, it should be noted that our meta-analysis was limited by a small number of patients included with a significant risk of biases in the majority of the included randomized controlled trials.

Mortality benefits elicited with the use of ivermectin in patients with COVID-19 likely stem from the ability of 


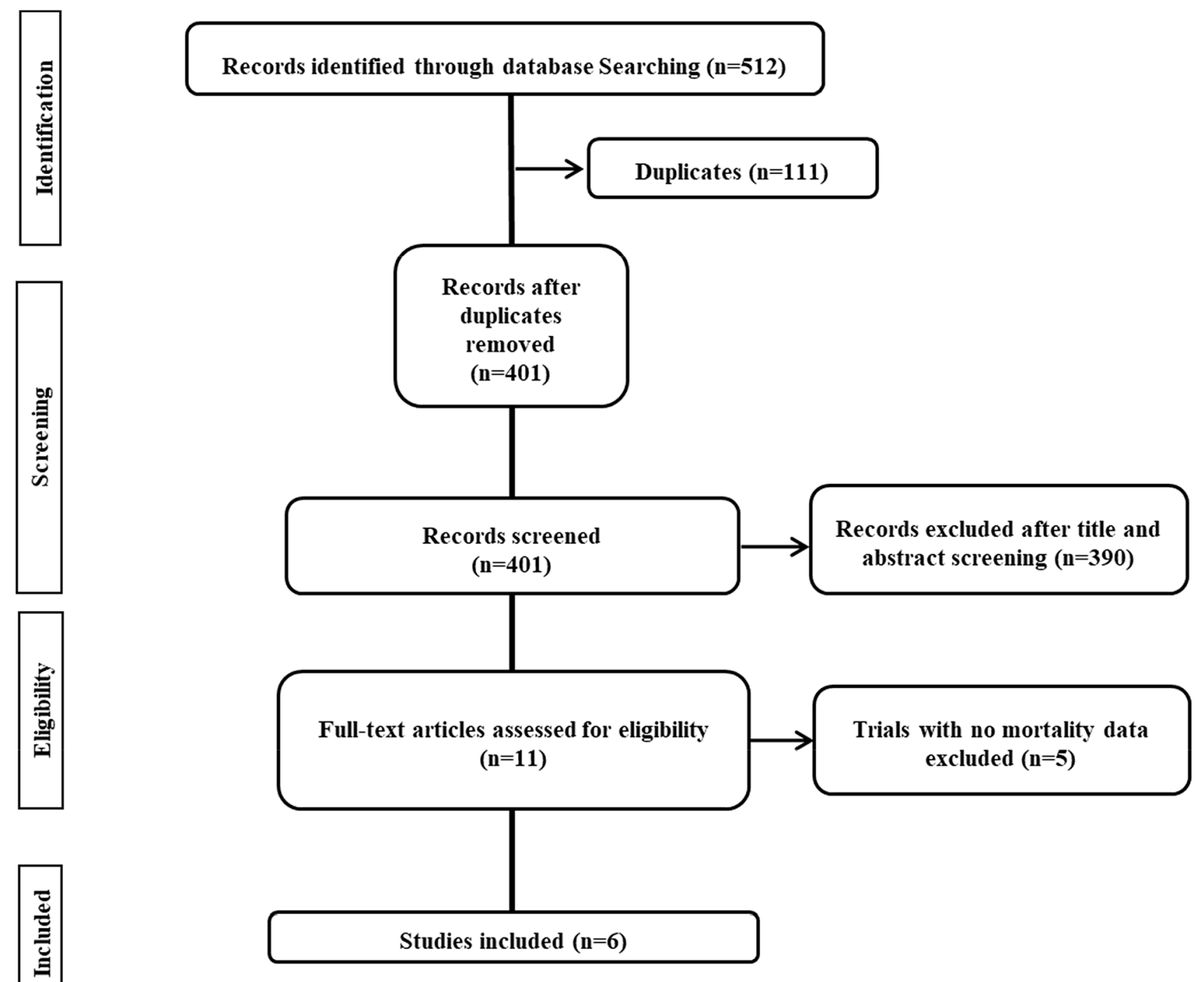

Fig. 1 PRISMA (Preferred Reporting Items for Systematic Reviews and Meta-analyses) flow diagram of process of study selection

ivermectin to hasten clinical recovery in which a higher proportion of patients achieved clinical improvement (inconsistently defined across the included trials) in the ivermectin group compared to the control group (60.7\% versus $44.4 \%$ in the trial by Mahmud et al. [7]; $96.5 \%$ versus $62.0 \%$ in the trial by Elgazzar et al. [8]; and $46.7 \%$ versus $36.7 \%$ trial by Okumuş et al. [9]). Besides, ivermectin could also prevent clinical deterioration in patients with COVID-19 wherein a lower proportion of patients deteriorated clinically (inconsistently defined across the included trials) in the ivermectin group compared to the control group $(4.3 \%$ versus $10.0 \%$ in the trial by Hashim et al. [5]; $8.7 \%$ versus $17.8 \%$ in the trial by Mahmud et al. [7]; and 2.5\% versus $26.0 \%$ in the trial by Elgazzar et al. [8]).

Noteworthily, the use of hydroxychloroquine in the treatment arm in the trial by Niaee et al. [6] may have confounded study outcomes. The trial by Hashim et al. [5] also offered doxycycline along with ivermectin in the intervention arm along with the standard care that also included azithromycin and corticosteroids (dexamethasone or methylprednisolone if needed). Similarly, the study by Elgazzar et al. [8] also included a complex treatment regimen where trial subjects also received azithromycin, vita$\min \mathrm{C}$, zinc, lactoferrin, acetylcysteine, and prophylactic or therapeutic anticoagulation in addition to ivermectin; this was compared with a controlled group that received hydroxychloroquine. There was a potentially higher risk of cardiac arrhythmia in the control group that may have confounded the higher mortality observed ( 2 deaths versus 24 deaths in treatment and control groups, respectively). Indeed, RECOVERY trial had previously demonstrated a greater risk of cardiac death in COVID-19 patients on 


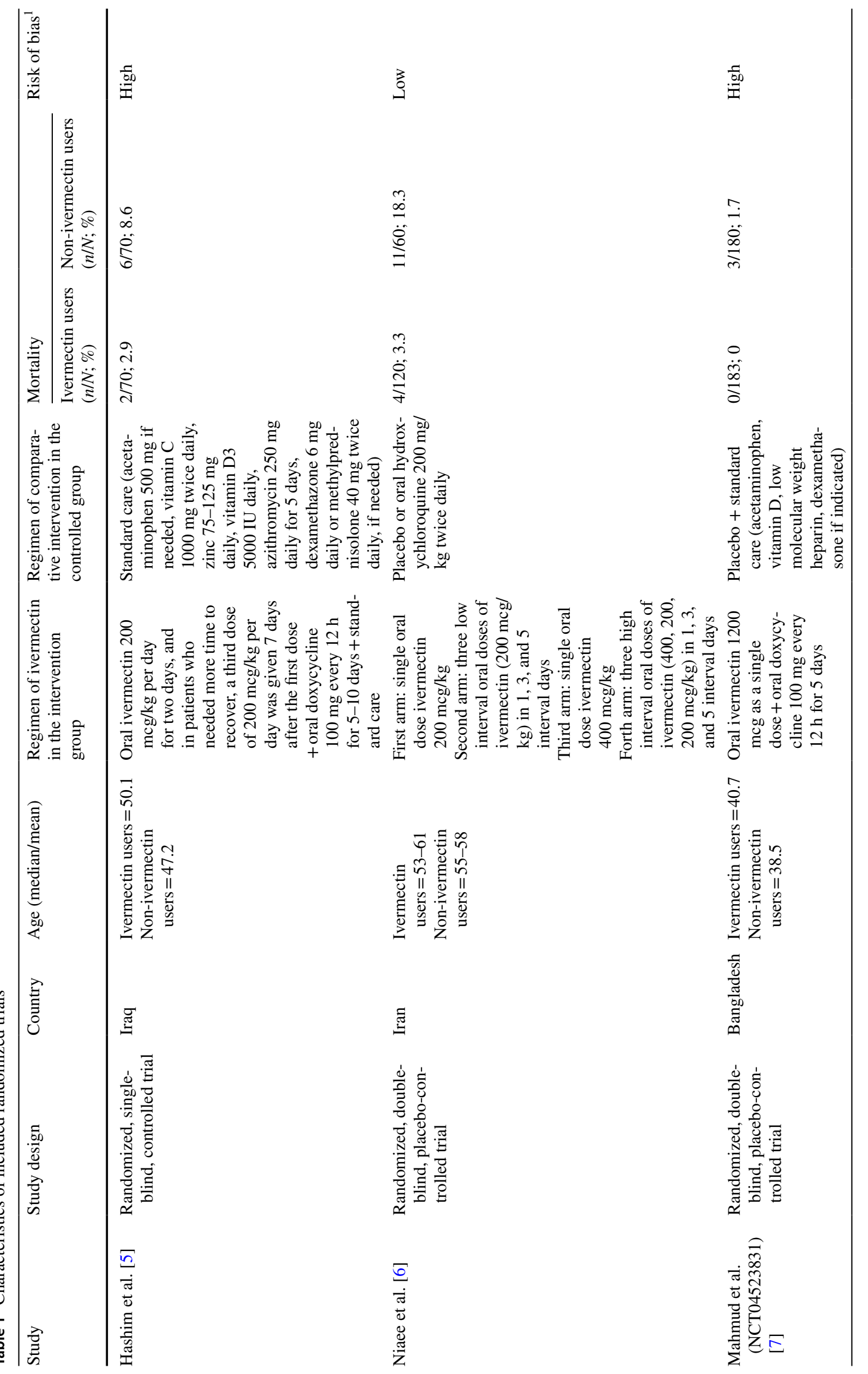




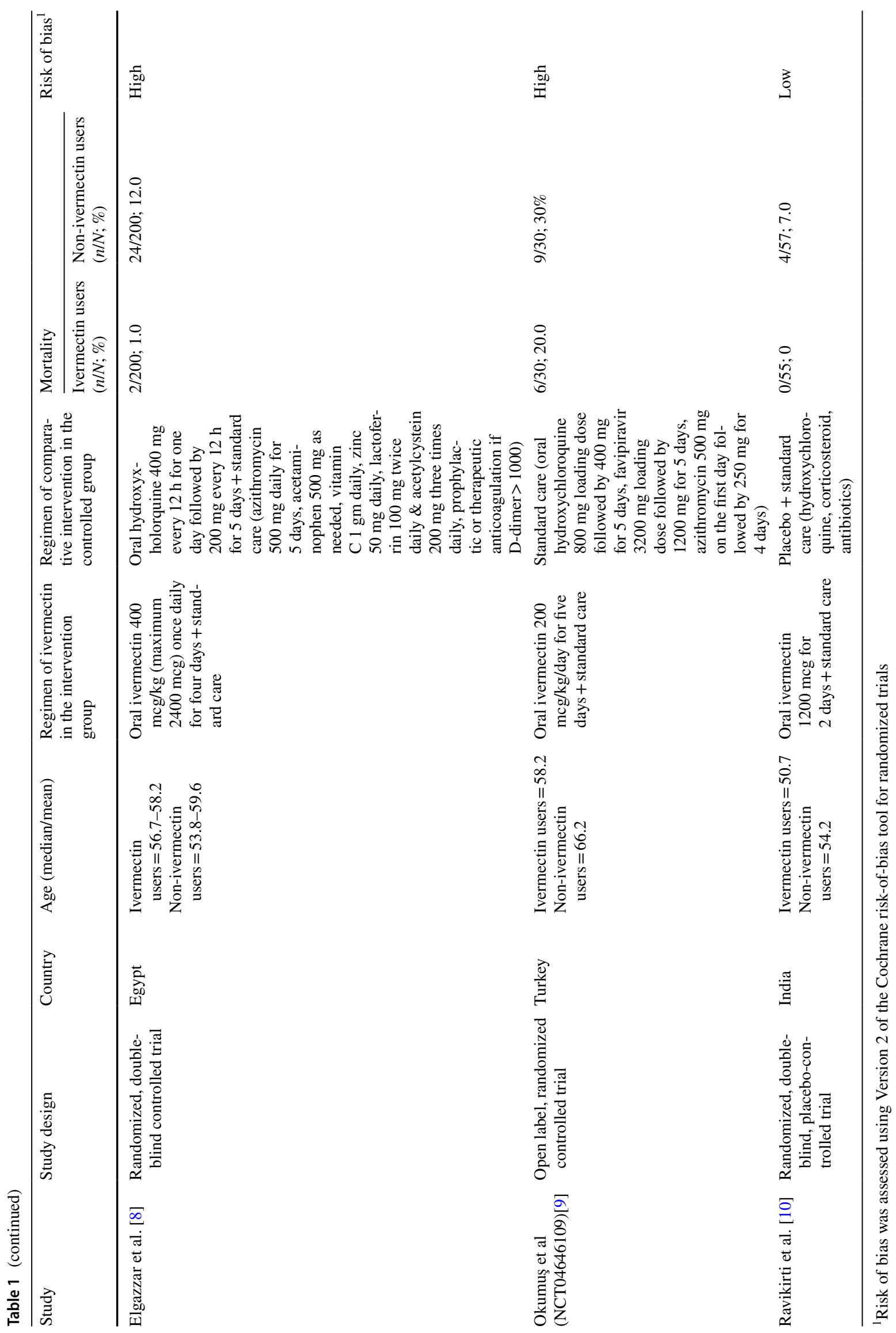




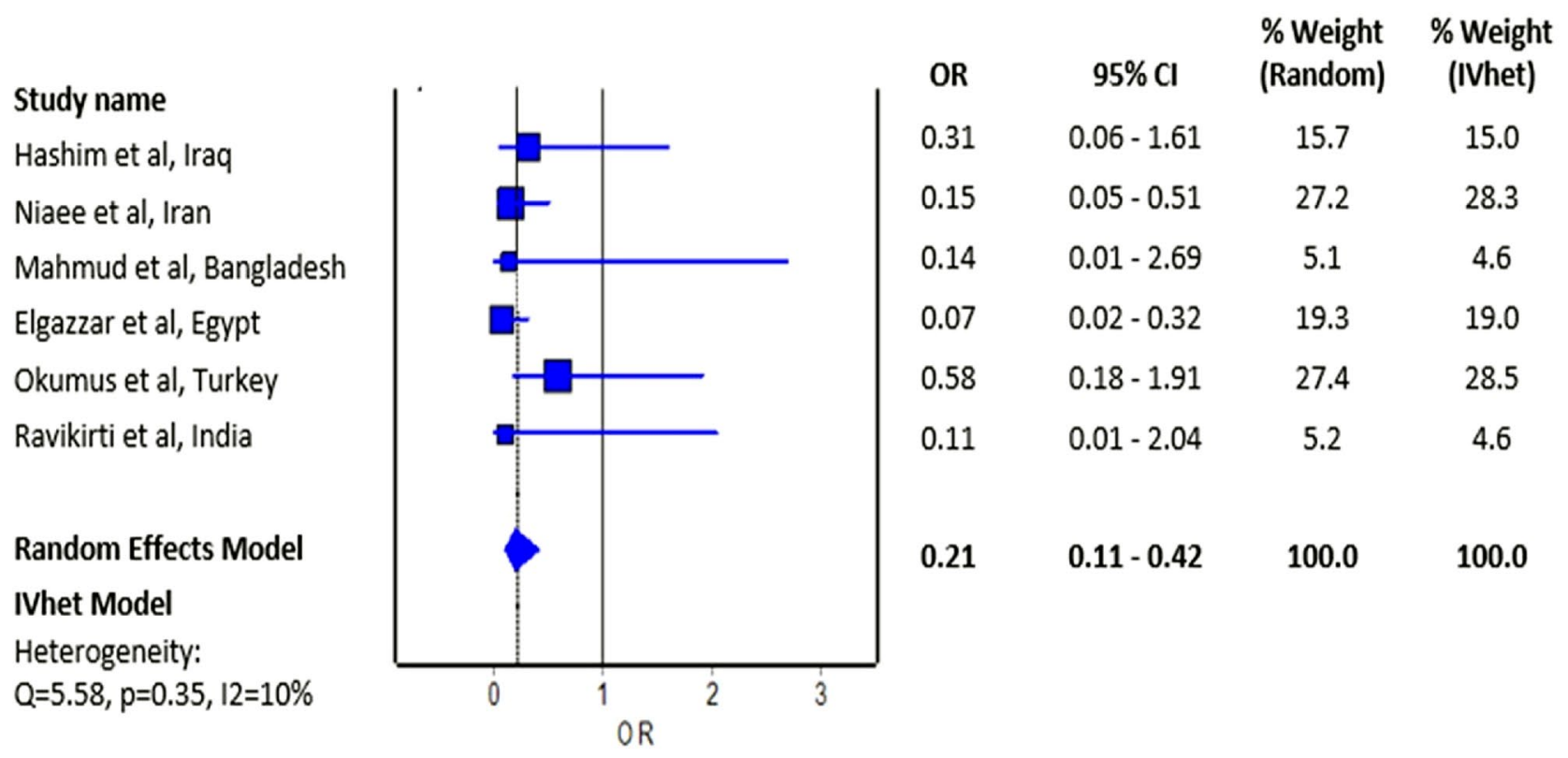

Fig. 2 Forest plot showing the pooled odds ratio of mortality between ivermectin users and non-ivermectin users with COVID-19

hydroxychloroquine [12]. On the other hand, the trial by Okumuş et al. [9] included hydroxychloroquine, favipiravir (a broad-spectrum antiviral drug), and azithromycin as part of the standard care in addition to ivermectin. It is worth mentioning that the randomized controlled trials by Niaaee et al. [6], Elgazzar et al. [8], and Okumuş et al. [9] contributed over $70 \%$ weight in this meta-analysis and therefore a more conclusive clinical evidence is still warranted to confirm the mortality benefits.

In addition, the reporting of adverse events in the included trials had been poor. Only two trials included the details on the safety of ivermectin in patients with COVID-19; the trial led by Mahmud et al. [7] reported erosive esophagitis in one patient in the treatment arm (ivermectin + doxycycline) but the use of doxycycline was likely the culprit, since doxycycline alone is one of the most common causes of druginduced esophageal ulcers [13]; the trial led by Okumuş et al. [9] reported nausea and vomiting in two patients and increased serum levels of liver enzymes in one patient, but without serious adverse events or treatment-related adverse events with ivermectin.

We were aware of at least one treatment protocol of COVID-19 [14] at the time of writing this manuscript that recommended the use of ivermectin in patients with COVID-19. Given the reported mortality benefits with ivermectin are still preliminary, we suggest that ivermectin should preferably be administered under clinical trial settings until warranted by more conclusive large-scale randomized controlled trials.
Funding No funding or sponsorship was received for the publication of this article.

\section{Declarations}

Conflict of interest All named authors declare that they have no potential conflict of interest.

\section{References}

1. Heidary F, Gharebaghi R. Ivermectin: a systematic review from antiviral effects to COVID-19 complementary regimen. J Antibiot (Tokyo). 2020;73(9):593-602.

2. Caly L, Druce JD, Catton MG, Jans DA, Wagstaff KM. The FDAapproved drug ivermectin inhibits the replication of SARS-CoV-2 in vitro. Antiviral Res. 2020;178:104787.

3. Moher D, Shamseer L, Clarke M, et al. Preferred reporting items for systematic review and meta-analysis protocols (PRISMA-P) 2015 statement. Syst Rev. 2015;4(1):1.

4. Sterne JAC, Savović J, Page MJ, et al. RoB 2: a revised tool for assessing risk of bias in randomised trials. BMJ. 2019;366:14898.

5. Hashim HA, Maulood MF, Rasheed, AM, Fatak DF, Kabah KK, Abdulamir AS. Controlled randomized clinical trial on using Ivermectin with Doxycycline for treating COVID-19 patients in Baghdad, Iraq. Preprint. medRxiv. 2020;https://doi.org/10.1101/ 2020.10.26.20219345.

6. Niaee MS, Gheibi N, Namdar P, et al. Ivermectin as an adjunct treatment for hospitalized adult COVID-19 patients: A randomized multi-center clinical trial. Preprint. Research Square. 2020; https://doi.org/10.21203/rs.3.rs-109670/v1.

7. ClinicalTrials.gov. Clinical Trial of Ivermectin Plus Doxycycline for the Treatment of Confirmed COVID-19 Infection. https://clini caltrials.gov/ct2/show/NCT04523831 
8. Elgazzar A, Hany B, Youssef SA, Hafez M, Moussa H. Efficacy and Safety of Ivermectin for Treatment and prophylaxis of COVID-19 Pandemic. Preprint. Research Square. 2020;https:// doi.org/10.21203/rs.3.rs100956/v1.

9. ClinicalTrials.gov. Ivermectin for Severe COVID-19 Management. https://clinicaltrials.gov/ct2/show/NCT04646109

10. Ravikirti, Roy R, Pattadar C, et al. Ivermectin as a potential treatment for mild to moderate COVID-19-A double blind randomized placebo-controlled trial. Preprint. 2021;2021.01.05.21249310.

11. Rajter JC, Sherman MS, Fatteh N, Vogel F, Sacks J, Rajter JJ. Use of Ivermectin Is Associated with Lower Mortality in Hospitalized Patients with Coronavirus Disease 2019: The ICON Study [published online ahead of print, 2020 Oct 13]. Chest. 2020;S0012-3692(20)34898-4.
12. RECOVERY Collaborative Group, Horby P, Mafham M, et al. Effect of Hydroxychloroquine in Hospitalized Patients with Covid-19. N Engl J Med. 2020;383(21):2030-2040.

13. Zografos GN, Georgiadou D, Thomas D, Kaltsas G, Digalakis M. Drug-induced esophagitis. Dis Esophagus. 2009;22(8):633-7.

14. Marik P. EVMS COVID-19 Management Protocol: An overview of the MATH+ and I-MASK+ Protocols. https://www.evms.edu/ media/evms_public/departments/internal_medicine/EVMS_Criti cal_Care_COVID-19_Protocol.pdf

Publisher's Note Springer Nature remains neutral with regard to jurisdictional claims in published maps and institutional affiliations. 